\title{
The Patient-Centered Oncology Care on Healthcare Utilization and Cost: A Systematic Review and Meta-Analysis
}

\author{
Hailun Liang ${ }^{1}$, Lei Tao ${ }^{2}$, Eric W. Ford ${ }^{3}$, May A. Beydoun ${ }^{4,}{ }^{\star}$, Shaker M. Eid ${ }^{5}$ \\ ${ }^{1}$ Hailun Liang, DrPH, MSc, Research Associate, Department of Health Policy and Management, \\ Johns Hopkins Bloomberg School of Public Health, Baltimore, MD, USA \\ 2Lei Tao, MSc, PhD Student, Institute of Public Policy and Public Finance, Renmin University of \\ China, School of Public Administration and Policy, Beijing, China \\ ${ }^{3}$ Eric W. Ford, PhD, MPH, Professor, Department of Health Care Organization and Policy, \\ University of Alabama at Birmingham, School of Public Health, Birmingham, AL, USA \\ ${ }^{4}$ May A. Beydoun, PhD, MPH, Staff Scientist, Laboratory of Epidemiology and Population \\ Sciences, National Institute on Aging, NIA/NIH/IRP, Baltimore, MD, USA \\ ${ }^{5}$ Shaker M. Eid, MD, MBA, Associate Professor, Department of Medicine, Johns Hopkins School \\ of Medicine, Baltimore, MD, USA
}

\section{Abstract}

Background-Optimal cancer care entails the coordination among multiple providers, continued follow-up and surveillance over time. The patient-centered care brings opportunities to improve the delivery of cancer care. The adoption of patient-centered oncology care (PCOC) is in its infancy. Evidence synthesis on the model's effectiveness is scant.

Purposes-This is the first systemic review and meta-analysis on associations of PCOC with cancer patients' adverse healthcare utilization, cost, patient satisfaction and quality of care.

Methods-Our study was guided by the Preferred Reporting Items for Systematic Reviews and Meta-Analyses (PRISMA) framework. Quality appraisal was performed using Downs and Black's quality checklist. Study-level effect sizes of adverse healthcare utilization were computed using Cohen's $D$ and summarized using forest plots. Funnel plots were constructed to examine publication bias.

Results-Of 334 studies that were reviewed, ten met eligibility criteria and were included into the final analysis. Many included studies implemented almost all six of patient-centered care core attributes, plus three additional attributes that specifically addressed cancer patients' needs, including triage pathways, standardized and evidence-based symptom management, as well as support patient navigation. PCOC patients had lower utilization of inpatient care (standardized means difference $[\mathrm{SMD}]=-0.027, \mathrm{P}=0.049)$. Overall positive effect of PCOC on emergency department use was small and not significant $(\mathrm{SMD}=-0.023, \mathrm{P}=0.103)$. In terms of cost and

*Corresponding author:May A. Beydoun, PhD, MPH, Biomedical Research Center, 251 Bayview Blvd., Suite 100, Room 04B118, Baltimore, MD 21224 Tel: 410-558-8648 Fax: 410-558-8236 baydounm@mail.nih.gov. 
quality of care, our narrative summaries showed an overall positive direction, though we found limitations in individual study quality that precluded a meta-analysis.

Public Implication-The results showed that it is possible to utilize patient-centered model to support best-practice of cancer care. Early evidence shows that the PCOC model has potential to improve healthcare utilization, cost and quality of care, but limited numbers of included articles and heterogeneity of those studies implied that more rigorous research is expected to further investigate the model's effects.

\section{Keywords}

patient-centered care; oncology; systematic review

\section{INTRODUCTION}

Cancer has a major impact on society worldwide and is the second leading cause of death in the United States. Currently, the number of patient living with a cancer diagnosis is projected to increase, it is mainly due to the increase in the incidence of cancer (National Cancer Institute 2018). The US has 15.5 million individuals living with a cancer diagnosis, a number projected to reach 19 million by 2024 (American Cancer Society, 2017). In 2017, it was estimated that 1,688,780 new cancer cases were diagnosed and 600,920 people died of cancer in the US (American Cancer Society, 2017). All these newly diagnosed cancer patients demand medical care at every stage of their cancer experiences. The Institute of Medicine (IOM)'s report identified six major phases in the cancer care continuum: prevention, early detection, diagnosis, treatment, survivorship, and end-of-life care (Hewitt et al., 2005). The greater intensity of health care service use by cancer patients trigger increasing health care cost, resulting in a greater overall burden of cancer. It is estimated that the direct medical costs for cancer in the US in 2014 were $\$ 87.8$ billion (American Cancer Society, 2017). Besides mounting cancer incidence rates, an increasing cost of medications and technologies, the excessive utilization of inpatient and emergency services coupled with fragmented care delivery are all contributing to the overall burden of cancer care (Ahmed et al., 2015).

Optimal and cost-effective cancer care entails the coordination of efforts from multiple providers, the use of several procedures and medications, coupled with continued follow-up and surveillance over time. Moreover, many health conditions are associated with cancer. For example, people age 65 and older are more likely to have one or more chronic health problems in addition to living with cancer at all stages, which include high blood pressure, heart disease, diabetes, and arthritis, etc (National Cancer Institute 2016). With increased disease burden, it requires innovative patient-management model and alternative payment reforms (Edwards et al., 2014; Wilper et al., 2008). Placing emphasis on team-based care, care coordination, patient-centered orientation, enhanced access to care, and quality improvement (Arend et al., 2012), the patient-centered medical home (PCMH) model brings opportunities to evolve and improve the delivery of cancer care. The PCMH has emerged as a solution to fragmented care delivery, which was developed to create a strong physicianpatient relationship that results in more personal, coordinated, effective, and efficient care (National Committee for Quality Assurance [NCQA], 2011). PCMHs models have been 
applied broadly in different clinical settings and to different types of patients and settings, including in community health centers, chronic disease patients, and low-income or medically underserved populations (Shi et al., 2017; Hoff et al., 2012; Liang et al., 2017). A number of studies and literature reviews have shown that PCMHs are effective in improving patients' outcomes, quality of care as well as reducing costs among general populations (Arend et al., 2012; Hoff et al., 2012; Jackson et al., 2013; Reibling, 2016). Two recent systematic reviews also concluded that PCMH adoption was effective among specific population sub-groups, such as low-income and diabetic patients (van den Berk-Clark et al., 2017; McManus, 2017).

As health reform efforts to improve quality and experience of care move forward, PCMH models are incorporated into more health care settings, preferring value over volume and redesigning the entire health care delivery system (Huang and Rosenthal 2014). For example, building on the success of the PCMH, the NCQA announced a Patient-Centered Specialty Practice (PCSP) recognition program in 2013, which is a practice-based evaluation for clinicians who provide care in non-primary care specialties. It puts patients first, requires providers to organize care around patients—across all clinicians seen by a patient—and to include patients in planning care and as partners in managing conditions (NCQA 2013). The PCSP program is valuable for building delivery system reforms that go beyond primary care, while strengthening and clarifying the roles of both primary care and specialty practices (NCQA 2013). Envisioning a patient-centered, triage pathway for the patients, potential advantages of PCSP models become clear. A practice with the PCSP recognition would have mechanisms in place to provide patients prompt access for clinical consultations. Primary care providers are involved at each step in the patient's care, and streamlined referrals could be well-coordinated. Patients would have seen a specialist in a timely manner and can focus more on their health needs rather than navigating the health system. Redundant testing and unnecessary medical procedures can be avoided (NCQA, 2013). One example of such specialty practices is the adoption of patient-centered models in cancer treatment. The NCQA, in conjunction with the American Society of Clinical Oncology (ASCO), the RAND Corporation, National Coalition for Cancer Survivorship, and Independence Blue Cross, was piloting a patient-centered oncology care (PCOC) model within a Patient-Centered Outcomes Research Institute-funded project in southeastern Pennsylvania. Early evidence showed these oncology practices in Pennsylvania successfully demonstrated that implementation of a PCSP-like model in cancer care. These efforts have the potential to bend the cost curve while also improving quality of care and patient satisfaction (Page et al., 2015).

One of the greatest challenges in cancer care is avoiding emergency department (ED) visits and unplanned hospitalizations during treatment and survivorship (American Cancer Society, 2013). Moreover, managing cost and quality for oncology care is also challenging. Innovative patient-management models in oncology based on PCMH principles, coupled with alternative payments, such as bundled payment and pay-for-performance, showed evidence of effectiveness, with bended curve of cost, improved quality of care and enhanced patient outcomes (Page et al., 2015; Eagle and Sprandio 2012; Collins et al., 2010). The PCOC model represents a logical platform on which to build a more comprehensive valueoriented, evidence-based cancer care delivery system. 
The adoption of PCOC is in its infancy. Importantly, systematic review and evidence synthesis on the model's effectiveness is scant. Our present study will address this knowledge gap and is particularly timely given the recent launch of the Centers for Medicare and Medicaid Services (CMS) Oncology Care Model. The latter is a new payment and delivery model incorporating elements of care coordination, navigation, and national treatment guidelines for cancer care (CMS, 2016). Evidence from our study can inform the implementation of PCOC.

\section{METHODS}

\section{Search Strategy}

Comprehensive search strategies were applied during the electronic search of four databases: PubMed, Cochrane Library, EMBASE, and Google Scholar. The year of publication was set from January $1^{\text {st }}, 2007$ to May $31^{\text {st }}, 2018$. Our searching key words and MeSH terms include: "patient-centered medical home", "medical home", "patient-centered care (MeSH term)", "oncology medical home", "whole person orientation", "patient-focused care", and "delivery of health care, integrated" (MeSH term). We also filtered for "cancer", "neoplasms" (MeSH term) and "oncology".

\section{Definition of PCOC and Conceptual Framework}

In 2007, the major primary care physician associations endorsed the concept and Joint Principles of the PCMH, which was best described as a model or philosophy of care that is patient-centered, comprehensive, team-based, coordinated, accessible, and focused on quality and safety (Patient-Centered Primary Care Collaborative 2012). In 2011, the NCQA proposed the PCMH standards which include 6 core principles: (1) enhance access and continuity, (2) use data for population management, (3) care management, (4) support selfcare process, (5) referral tracking and follow-up, (6) implement continuous quality improvement (NCQA 2011). In this study, we adopted the above definition and principles, and constructed a conceptual framework to guide this study (Figure 1). The framework represents a heuristic for understanding of the demonstrated linkages between PCOC interventions and specific health care outcomes. As presented in the Figure 1, the conceptual framework for PCOC is centered around six PCMH dimensional constructs and three oncology-specific dimensional constructs that may lead to expected outcomes (PatientCentered Primary Care Collaborative 2012; Wender and Altshuler 2009). For example, the dimension of identifying and managing patient population may help to facilitate care of less activated patients, such as assessing and documenting patient risk factors, and therefore identifying patients for proactive reminders. The other mechanism is that PCOC supports patients by establishing relationships with providers involved in their care during active treatment and connecting patients with community and financial resources (Arend et al., 2012; Jackson et al., 2013). Strong physician-patient relationships make care more coordinated, effective, and efficient (Hoff et al., 2012; Reibling, 2016). Moreover, under PCOC model, practices systematically record patient information for patient populations and uses it to coordinate patient care (NCQA 2011). 
In terms of three additional components related to oncology care, they were extracted based on the synthesized evidence from CMS on key drivers for improving oncology care (CMS 2017). The three additional components extend current conceptualization of PCMH, and particularly target oncology outcomes and the delivery of quality and value in cancer care (ASCO 2014; CMS 2017). In terms of triage pathway, the PCOC practices may identify and implement pathways for disease management that address common side effects experienced by its patient population. Besides, pathways are used in the triage process to ensure health problems are addressed appropriately and proactively managed to prevent unnecessary ER visits and impatient utilization (Sprandio, 2010; Eagle and Sprandio 2012). For standardized and evidence-based symptom management, the PCOC practices may adopt evidence-based algorithms or tools for appropriate diagnosis, treatment and symptom management. These tools can be electronic or manual, but should be used systematically to ensure a standardized approach to providing evidence-based cancer care (Page et al., 2015; Kuntz et al., 2014). With respect to patient navigation support, it helps remove any roadblocks to treatment, so that patients and their families are able to find and make use of the various resources, treatment services, and support services that they need to receive the best cancer treatment possible (Kohler et al., 2015; Wheeler et al., 2013). If these above dimensions work in the intended manner, then expected outcomes would include lower utilization of high-cost care, cost savings and better quality of care (Patient-Centered Primary Care Collaborative 2012; Wender and Altshuler 2009; Rosenthal 2008).

\section{Study Selection}

Initial records were identified from four electronic databases. Figure 2 outlines the screening process. Using the reference management software Endnote 8.0, duplicates were excluded automatically. Next, two independent investigators read the title and abstract of the related articles and assessed them according to the following selection criteria: 1) the intervention is a PCMH model that meets the six PCMH dimensional constructs in the conceptual framework; 2) research design is randomized controlled trial, cohort study, case control study, or case series; 3 ) stages of cancer care include any stages; 4) intervention is conducted at any types of setting (such as primary care and specialists); 5) conducted in the United States.

Articles that met these criteria or could not be clearly identified through the title and abstract screening would be kept for the next screening stage. Any disagreements were resolved through discussion between the two investigators (HL and LT). Through this stage, studies were excluded, remaining studies to full-text screening. Two investigators read the full texts of the remaining articles to assess them according to the following inclusion criteria:1) the model meet the core principles of the PCMH 2) the participants are cancer patients 3) the purpose or outcomes is focused on healthcare utilization, cost, patient satisfaction and quality of care. Finally, eligible articles were finalized and data were extracted. We extracted data on author, publication year, study design, participants, description of the intervention, types of outcome, results and quality index. Table 1 describes the above characteristics of each articles. 


\section{Quality Assessment}

Two investigators independently assessed the quality of the included studies based on the Downs and Blacks Checklists. The checklists contain a total of 27 items in 5 sections. Each item is given based on the answer to a detailed score, the higher the final score, the higher the quality of the literature, with an overall score of 33. Accordingly, the quality of the studies is divided into three levels based on the final score: low quality (1-11), medium quality (12-22), high quality (23-33). Disagreements about the final score were solved by two investigators through consultation.

\section{Data Synthesis and Analysis}

All analyses were conducted using Stata 12.0 (StataCorp, 2011). We extracted the outcomes into three main categories: 1) the utilization of care (emergence room [ER] visits, inpatient visits, and outpatient visits); 2) cost of care; 3) the quality of care and patient satisfaction. Study-level effect sizes of adverse healthcare utilization were computed using Cohen's $D$ with the Bessel's correction and summarized using forest plots. We used a random effects model. The heterogeneity in the meta-analysis was assessed using Cochran Q test and $\mathrm{I}^{2}$ statistics. Funnel plots and Egger's test were constructed to examine publication bias. A funnel plot is a simple scatter plot of the intervention effect estimates from individual studies against measure of each study's size or precision. Using Egger's test, P value was calculated by a linear regression of intervention effect estimate against its standard error, weighted by the inverse of the variance of the intervention effect estimate (Egger, 1997). Other outcomes such as quality of care and patient satisfaction, cost of care and outpatient visits for reviews where the nature of data were diverse and not feasible to be extracted for quantitative syntheses. We summarized characteristics of studies in the Table 1 and provide narrative descriptions for each included study. The Preferred Reporting Items for Systematic Reviews and Meta-Analyses (PRISMA) framework guided the conduct and reporting of this study (Moher et al., 2009).

\section{RESULTS}

\section{Results of Search}

We initially identified 3,158 citations based on our search criteria and obtained 25 citations from other sources. As described in Figure 2 (PRISMA form), there were 2,038 remaining titles after removing duplicates. We reviewed the titles and abstracts for inclusion and 1,704 were excluded as not meeting the criteria. Those excluded before obtaining full text papers were studies that were not original research studies, unrelated to patient-centered care, and were not applied to the treatment for cancer patients. We reviewed full text of 334 articles as broadly relevant. Of these, 324 were excluded, leaving a total of 10 articles. Those assessed in full text and excluded were ineligible in study designs, or were about a single patientcentered care intervention rather than the PCMH model as described above.

\section{Study Characteristics}

The final included articles consisted six cohort studies (Sullivan et al. 2018; Colligan et al., 2017a; Colligan et al., 2017b; Kohler et al., 2015; Goyal et al., 2014; Wheeler et al., 2013) 
and four case series and case reports (Waters et al., 2015; Harper et al., 2015; Kuntz et al., 2014; Sprandio, 2010). The Table 1 summarized the characteristics of the included studies. Patients were identified in the studies using International Classification of Disease, ninth edition (ICD-9) codes indicating active cancers. Among the ten included articles, the types of cancer included breast, lung, colorectal, lymphoma, pancreatic, thyroid, melanoma, male or female genitourinary cancers, or head or neck cancers in the analytic sample. Included studies mainly compared outcomes between cancer patients who received PCOC and usual type of care. Data sources included cancer registry data, claims data, chart and other administrative data, as well as self-report/survey data. Patients included patient who received care at physician office, hospitals, specialist, or oncology practice.

\section{Study Quality Appraisal}

We used the Downs and Black's Quality Index (Downs and Black, 1998) to assess the quality of each study. Based on the quality of reporting, internal validity (bias and confounding), study power, as well as external validity, an overall score and a level of quality appraisal was given to each study. As shown in Table 1, we scored three studies as high quality (scored above 23), and seven studies as medium quality (scored range from 12 22). Of those six cohort studies, three were scored as medium quality. All four studies for narrative summaries were graded as medium quality. In terms of major factors led to medium quality, internal bias and selection bias, such as lack of attempt made to blind study subjects, and incomprehensive adjustment for confounding in the analyses, were concluded in most studies with medium quality appraisal.

\section{Attributes of Patient-Centered Oncology Care}

Most studies appeared to implement the majority of six PCMH core attributes. Moreover, these included oncology care models addresses the extra steps involved in using a patientcentered approach to treat patients with cancer. We found that besides the core component of PCMH, three additional components related to oncology care were commonly implemented by most included studies, which were (1) telephone triage pathways to help first responders identify patient symptoms and ensure patients receive appropriate care (trained nurses utilized customized symptom management algorithms to ensure health problems were addressed appropriately and proactively managed to prevent unnecessary health service utilization) (Sullivan et al. 2018; Colligan et al., 2017a; Colligan et al., 2017b; Waters et al., 2015; Harper et al., 2015; Kuntz et al., 2014; Sprandio, 2010); (2) standardized and evidence-based symptom management (Colligan et al., 2017a; Colligan et al., 2017b; Kohler et al., 2015; Goyal et al., 2014; Kuntz et al., 2014; Sprandio, 2010); and (3) support patient navigation (Sullivan et al. 2018; Colligan et al., 2017b; Kohler et al., 2015; Goyal et al., 2014; Wheeler et al., 2013; Sprandio, 2010), such as coordinating referrals and assisting with transitions across settings and providers.

\section{Adverse Healthcare Utilization}

Six out of ten studies reported the outcomes of ED use (Colligan et al., 2017a; Colligan et al., 2017b; Goyal et al., 2014; Kohler et al., 2015; Kuntz et al., 2014; Sprandio, 2010). Negative effect sizes denoted improvement when it came to ER utilization. Negative effects are found in three of four analytical studies (Colligan et al. 2017a; Colligan et al. 2017b; 
Goyal et al. 2014), and only one study showed positive effect (Kohler et al., 2015) but with non-statistical significance. The meta-analyses were performed with a random effects model for assessing heterogeneity. The results of $\mathrm{I}^{2}$ represented moderate heterogeneity ( $\mathrm{I}^{2}$ ranging $30 \%$ to 50\% may represent moderate heterogeneity), however, the $\mathrm{P}$ value indicated that the evidence of heterogeneity of intervention effects was not statistically significant $\left(\mathrm{I}^{2}=\right.$ $41.24 \%, \mathrm{P}=0.17)$. Overall effect size of four analytical studies was small and not statistically significant (standardized means difference $[\mathrm{SMD}]=-0.023, \mathrm{P}=0.103$ ). The highest effect was in Goyal et al. (2017a, SMD $=-0.083, \mathrm{P}=0.001$ ). Publication bias was assessed by funnel plots and Egger's tests. The results showed that there was no statistical evidence of publication bias in the meta-analysis $(\mathrm{P}=0.491)$. Among the two studies for narrative summaries, Kuntz et al. (2014) found the ER visits were lower among the patients who enrolled in the oncology medical home. Similarly, in Sprandio's study (2010), the ER referrals had decreased progressively since patients participated the oncology PCMH.

Six studies assessed the inpatient use (Colligan et al., 2017a; Colligan et al., 2017b; Goyal et al., 2014; Kohler et al., 2015; Kuntz et al., 2014; Sprandio, 2010). Negative effect sizes denoted improvement when it came to inpatient utilization. The negative effects were found in three (Colligan et al., 2017a; Goyal et al., 2014; Kohler et al., 2015) of four analytical studies (Colligan et al., 2017a; Colligan et al., 2017b; Goyal et al., 2014; Kohler et al., 2015). The only one study with positive effects (Colligan et al., 2017b) was not statistically significant. The overall effect size of four analytical studies was statistical significant $(\mathrm{SMD}=-0.027, \mathrm{P}=0.049)$. The results of $\mathrm{I}^{2}$ represented substantial heterogeneity $\left(\mathrm{I}^{2}\right.$ ranging $50 \%$ to $90 \%$ may represent substantial heterogeneity), however, the $\mathrm{P}$ value indicated that the evidence of heterogeneity of intervention effects was not statistically significant $\left(\mathrm{I}^{2}=72.57 \%, \mathrm{P}=0.228\right)$. Publication bias was assessed by funnel plots and Egger's tests, and there was no statistical evidence of publication bias in the meta-analysis $(\mathrm{P}=0.80)$. Of the two studies for narrative summaries, Kuntz et al. (2014) found the numbers of the inpatient admissions were lower for patients who enrolled in the Michigan Oncology Medical Home Demonstration Project (MOMHDP), and Sprandio (2010) found a 16\% reduction in overall hospital admissions since patients participated the oncology PCMH. Moreover, two studies reported outpatient use (Goyal et al., 2014; Kohler et al., 2015). Goyal et al. (2014) found nonsignificant association between PCMH enrollment and the outpatient visits. On the contrary, Kohler et al. (2015) found PCMH enrollment was associated with 0.53 more outpatient visits monthly.

\section{Cost}

Four studies reported the costs (Colligan et al., 2017a; Colligan et al., 2017b; Kohler et al., 2015; Kuntz et al., 2014). In general, patients who were involved in PCMH model cost less compared to their comparative, historic or other groups. Colligan et al. (2017a) found that the cost of the oncology medical home model was associated with a lower Medicare cost of $\$ 675$ per patient per quarter through up to 3 years after the enrollment of the model $(\mathrm{P}<0.01)$. In Colligan et al. (2017b), the oncology medical home was associated with a decreased total Medicare cost of $\$ 3,346$ in the last 90 days of life. In Kuntz et al. (2014), the oncology medical home reduced an average estimated cost savings of $\$ 550$ per patient at the practices that participated in the first year of the demonstration project. However, there is 
one exception, showing a higher cost. Kohler et al. (2015) found that in the first 15 months after diagnosis, enrollment in a PCMH was related to an increase of \$429 in monthly average Medicaid spending.

\section{Quality of Care and Patient Satisfaction}

One included study reported the results of access of care (Wheeler et al., 2013), one included study reported the quality of end-of-life care (Sullivan et al., 2018) and two studies reported the patient satisfaction (Waters et al., 2015; Harper et al., 2015). In Wheeler et al. (2013), PCMH enrollment was significantly and positively related to the access of mammography $(\mathrm{P}=0.021)$. Waters (2015) found that patients were highly satisfied with the care they received in the PCMH project. Harper et al. (2015) found that patient satisfied with care in oncology medical home and felt comfortable with their plan of care.

\section{DISCUSSION}

We reviewed studies evaluating PCMH models applied to oncology care in order to better understand the essential component of PCOC, whether their adoptions were associated with lower rates of ER visits and hospitalization, whether they were related to reduced health care costs, and whether they were helpful in improving patient experience. We found that PCOC patients had lower utilization of inpatient care. Overall positive effect of PCOC on ER use was small and not significant. In terms of cost and quality of care, our narrative summaries showed an overall positive direction, though we found limitations in individual study quality that precluded a meta-analysis.

Compared with previous reviews, our findings may draw different conclusions related to the utilization measures. For example, a systematic review in 2013 on PCMH showed no effect on inpatient utilization among older adults with chronic conditions based on the results from five trials (Jackson et al., 2013). However, a recent systematic review and meta-analysis showed PCMH patients had lower utilization of emergency rooms (van den Berk-Clark, et al., 2017) among low-income population. This may have implied that the patient-centered care model may have different effects among different populations with varying diseases (Kohler et al., 2015).

Our study also demonstrated that many of the core components of PCOC were being implemented across studies. Serving as decision support tools, the triage pathways help trained responders, such as nurses or physician assistants, to ask questions and use symptom management algorithms to determine if a cancer patient is having an emergency, if the patient should be seen for a same-day appointment, or should be given phone advice and scheduled for a follow-up visit (Colligan et al., 2017a; Colligan et al., 2017b; Sprandio, 2010). For example, an included study by Sprandio (2010) found that more than $75 \%$ of all calls from triage system resulted in managing symptoms at home. About $10 \%$ resulted in a same-day visit and less than 5\% resulted in an ER visit. Another component applied among most included studies were standardization of diagnosis and treatment pathways in compliance with evidence-based guidelines. Many current studies showed that clinical process standardization is not only a driver of value, but also a major driver of higher efficiency, better quality of care and health outcomes (Page et al., 2015; Kuntz et al., 2014; 
Waters et al., 2015). The last component of patient navigation generally has a broad scope; navigation services were provided in and between multiple settings, including hospitals, ERs, and patient's home. This component showed promise in reducing adverse health care utilization among cancer patients (Colligan et al., 2017a).

Also of interest are mixed outcomes which were observed when it came to utilization and cost-saving. Specifically, there was a small improvement in ER use. Compared with patients who received usual care, patients who received PCOC were more likely to get enhanced access to the clinic or navigator by the use of the triage system, and could take advantage of extended and weekend hours, which helped them get timely care and avoid unnecessary ER visits (Colligan et al., 2017a). There was improvement when it came to inpatient visits. Our study reported statistically significant findings in decreased inpatient admissions among patient-centered oncology patient group. Many avoidable inpatient admissions are rooted in difficulties that patients have accessing timely care in the primary care setting. Our findings suggested an important potential value of patient-centered care's efforts to improve coordination of cancer care (Goyal et al., 2014).

In terms of cost, our narrative summaries showed an overall positive direction in costsavings. Among four studies reporting on health care spending, two cohort studies and one case series demonstrated reduced cost among PCOC groups, compared to the control groups. The key to controlling health care cost is the reduction and avoidance of unnecessary utilization and costs. Components of PCOC, such as patient triage and standard clinical pathways, can play an important role in shepherding patients through decision making and evidence-based symptom management to prevent unplanned hospitalizations and unneeded ER visits (Colligan et al., 2017a; Colligan et al., 2017b; Kuntz et al., 2014). Additionally, under the PCOC, successful management of the financial risks, and maximizing global patient management based on appropriate accredited benchmarks can make oncology practices remain viable going forward (McAneny, 2013; ASCO, 2014).

Included studies had some substantial limitations. First of all, because PCOC models are composed of a "basket" of multiple features, and definitions for components were different among models, examining and standardizing model components is beyond the scope of this work. Second, the included studies using a cohort design did not achieve random assignment, which hindered the possibility to conclude causal inferences. However, utilizing randomization design in social and health service studies may bring out ethical issues.

Future studies may choose an alternative strategy for overcoming this, such as one included study using propensity score matching, to control variables that could confound the exposure (Colligan et al., 2017a). Third, most included studies were based on the claims data and matched to cancer registry, which could cause a lack of reliable measures for controlling for cancer severity and stage. Fourth, due to PCOC at an early stage and limited number of literature on PCOC available, we did not set specific criteria for stages of cancer and types of settings. Future study may focus on the impact of PCOC on a specific stage of cancer care or within one type of setting where the interventions were implemented. Last, other factors may be associated with outcomes. Stratification involving the division of participants into subgroups with respect to categorical prognostic factors may use for a pooled estimate calculated across strata. However, the poor resolution in the measurement of confounders, 
e.g. co-morbidity assessed on a simple ordinal scale, precluded the possibility of conducting stratified analyses.

\section{PRACTICE IMPLICATIONS}

To our knowledge, this is the first systematic review and meta-analysis on patient-centered oncology care (PCOC). The findings of this systematic review offer further evidence for meaningful practice, and have many policy and research implications. In fact, the results from our study shed light on PCOC's ability to support best-practice of cancer care.

However, it is unclear whether the benefits gained from PCOC suggested by this review would offset challenges incurred by changes due to adoption of new models. For example, decreased hospitalization and increased outpatient use seems critical for strategic use of human and physical resources. Moreover, decreasing cost may translate to a reduced revenue to the health system. Moreover, allocating support and resources that are expected to help practitioners and administrators anticipate and respond to these changes due to the care model transition.

With regard to practice and policy implications, the lessons learned from the current synthesized evidence can further inform the implementation of these models. Many areas we identified as opportunities for improvement. From an administrator's perspective, although currently different types of PCOP models had many structures and processes in common, the PCOC practice standards varied widely across the practices. It is important to think broadly about how we can better use the main components of PCOC, and how we can make PCOP more standardized and documented. Some of the oncology-specific components were not established in a way that could produce documentation on demand. Besides, there were other areas, such as resources, infrastructure, leadership, cost vs. benefit that need to be considered for implementing PCOC. These areas are facilitators for practice transformation. For example, infrastructure for health information exchange and care coordination across practices was needed to improve professional interactions and patient care transitions. Moreover, the PCOC model should be scaled up in more health care institutions that should be chosen to represent the diversity of the cancer treatment facilities (i.e., community hospitals, teaching hospitals, comprehensive cancer centers).

It is important to also recognize the advancement of currently implemented patient-centered oncology models. The patient-centered models started being applied to oncology care and its further use has been encouraged by the recent launch of multiple care models across the nation. For examples, MOMHDP (Kuntz et al., 2014) and Community Oncology Medical Home (COME HOME) (Colligan et al., 2017a; Colligan et al., 2017b), have been widely adopted statewide or even nationwide, which implied these models' capacities of scaling up and sustainability. Our findings are very relevant to the CMS Oncology Care Model, which provides episode-based payments to practices for Medicare beneficiaries undergoing therapy, with quality performance accountability (CMS, 2016b). Under this model, participating practices are required to redesign themselves to include several aspects of PCOC (CMS, 2016a). Similar to PCOC, practices in the Oncology Care Model will provide therapies consistent with nationally recognized guidelines, and ensure timely access to appropriate care providers with real-time access to medical records (CMS, 2016b; Clough 
and Kamal, 2015). Moreover, these practices will use patient navigation to assist with transitions between health care settings and coordinating referrals (CMS, 2016b). PCOC offer valuable lessons on how to help patients avoid unnecessary utilization and thus reducing costs. The practices in the Oncology Care Model could benefit from adapting the PCOC's approach to design and implement core services to their context. On the other side, the Oncology Care Model provides an opportunity to test elements of the PCOC interventions on a larger scale, and with reformed payment mechanisms that could potentially support their increased implementation (Colligan et al., 2017b; Kline et al., 2015).

However, the currently available evidence is limited and in an earlier stage, more studies and evaluations are needed to determine the impact of the models on health care utilization, patients' health outcomes as well as cost. It is also crucial for collaborating to develop a core set of PCOC standards to enhance the focus, minimize the confusion, and shorten the timeline for adoption of new models. These unifications will enhance adoptability, and therefore the scalability of current models.

This review demonstrates that PCOC can be helpful with cancer patients, but the small number of relevant selected articles and the heterogeneity of those studies show that more rigorous research is expected to further investigate the model's effects on cancer patient care. It is important to use real-world evidence to support value in care delivery models. Providing high-quality care for cancer patients is challenging for several reasons, including the coordination of efforts from multiple providers, the use of several procedures and medications, coupled with continued follow-up and surveillance over time. The importance of innovative interventions and care models that can overcome these challenges, facilitate optimum care of cancer patients, and maintain quality of care given the increasing demand for care and shortages of providers. It has also been noted over time that, service, quality, and resource stewardship increases and will be further strengthened when considering the interrelationships among the three major entities in the service triad: the health care facilities, the providers, and the patient, as well as needs associated with health care performance. Reforms and policies should reflect these efforts, and the provision of such resources will promote the success of a sustainable transitions of cancer care. Given the emerging and promising evidence that PCOC is equipped to provide care management and coordination and improve communication, the authors of this review support that PCOC's role in addressing current challenges of delivering high-quality cancer care.

\section{Acknowledgments}

Conflicts of Interest and Source of Funding: This work was fully supported by the Intramural Research Program, National Institute on Aging, NIA/NIH/IRP. No conflict of interest declared

\section{References}

Ahmed S, Shahid RK, \& Gesy K (2015). Cancer care burden: aiming at the Achilles heel. Current Oncology, 22(3), e134-138. doi:10.3747/co.22.2252 [PubMed: 26089722]

American Cancer Society. (2017). Cancer Facts \& Figures 2017. Atlanta: American Cancer Society.

American Cancer Society. (2013). Economic impact of cancer. Retrieved from www.cancer.org/cancer/ cancerbasics/economic-impact-of-cancer. 
American Society of Clinical Oncology. (2014). Potential approaches to sustainable, long-lasting payment reform in oncology. Journal of Oncology Practice, 10(4), 254-258. doi:10.1200/ JOP.2014.001267 [PubMed: 24737874]

Arend J, Tsang-Quinn J, Levine C, \& Thomas D (2012). The patient-centered medical home: history, components, and review of the evidence. Mount Sinai Journal of Medicine, 79(4), 433-450. doi:10.1002/msj.21326 [PubMed: 22786733]

Centers for Medicare and Medicaid Services. (2016a). Oncology Care Model. Retrieved from https:// innovation.cms.gov/initiatives/oncology-care/.

Centers for Medicare and Medicaid Services. (2016b). Fact Sheet: Oncology Care Model. Retrieved from https://www.cms.gov/Newsroom/MediaReleaseDatabase/Fact-sheets/2016-Fact-sheets-items/ 2016-06-29.html.

Centers for Medicare and Medicaid Services. (2017). Key Drivers and Change Package. Retrieved from https: https://innovation.cms.gov/Files/x/ocm-keydrivers-changepkg.pdf.

Clough JD, \& Kamal AH (2015). Oncology Care Model: Short- and Long-Term Considerations in the Context of Broader Payment Reform. Journal of Oncology Practice, 11(4), 319-321. doi:10.1200/ JOP.2015.005777 [PubMed: 26060221]

Colligan EM, Ewald E, Keating NL, Parashuram S, Spafford M, Ruiz S, \& Moiduddin A (2017). Two Innovative Cancer Care Programs Have Potential to Reduce Utilization and Spending. Medical Care, 55(10), 873-878. doi: 810.1097/MLR.0000000000000795. [PubMed: 28906313]

Colligan EM, Ewald E, Ruiz S, Spafford M, Cross-Barnet C, \& Parashuram S (2017). Innovative Oncology Care Models Improve End-Of-Life Quality, Reduce Utilization And Spending. Health Affair (Millwood). 36(3), 433-440. doi: 410.1377/hlthaff.2016.1303.

Edwards BK, Noone AM, Mariotto AB, Simard EP, Boscoe FP, Henley SJ, . . Ward EM (2014). Annual Report to the Nation on the status of cancer, 1975-2010, featuring prevalence of comorbidity and impact on survival among persons with lung, colorectal, breast, or prostate cancer. Cancer, 120(9), 1290-1314. doi:10.1002/cncr.28509 [PubMed: 24343171]

Goyal RK, Wheeler SB, Kohler RE, Lich KH, Lin CC, Reeder-Hayes K, . . Mayer DK (2014). Health care utilization from chemotherapy-related adverse events among low-income breast cancer patients: effect of enrollment in a medical home program. North Carolina Medical Journal, 75(4), 231-238. [PubMed: 25046085]

Harper JL, De Costa AM, Garrett-Mayer E, \& Sterba KR (2015). Incorporating Patient Satisfaction Metrics in Assessing Multidisciplinary Breast Cancer Care Quality. Southern Medical Journal, 108(6), 372-376. doi: 310.14423/SMJ.0000000000000300. [PubMed: 26079465]

Hewitt ME, Greenfield S, Stovall E, \& National Cancer Policy Board (2005). From cancer patient to cancer survivor: lost in transition. Washington, DC: National Academies Press.

Hoff T, Weller W, \& DePuccio M (2012). The patient-centered medical home: a review of recent research. Medical Care Research Review, 69(6), 619-644. doi:10.1177/1077558712447688 [PubMed: 22645100]

Huang X, \& Rosenthal MB (2014). Transforming specialty practice--the patient-centered medical neighborhood. New England Journal Medicine, 370(15), 1376-1379. doi:10.1056/NEJMp1315416

Jackson GL, Powers BJ, Chatterjee R, Bettger JP, Kemper AR, Hasselblad V, . . Williams JW (2013). Improving patient care. The patient centered medical home. A Systematic Review. Annals of Internal Medicine, 158(3), 169-178. doi:10.7326/0003-4819-158-3-201302050-00579 [PubMed: 24779044]

Kline RM, Bazell C, Smith E, Schumacher H, Rajkumar R, \& Conway PH (2015). Centers for medicare and medicaid services: using an episode-based payment model to improve oncology care. Journal of Oncology Practice, 11(2), 114-116. doi:10.1200/JOP.2014.002337 [PubMed: 25690596]

Kohler RE, Goyal RK, Lich KH, Domino ME, \& Wheeler SB (2015). Association between medical home enrollment and health care utilization and costs among breast cancer patients in a state Medicaid program. Cancer, 121(22), 3975-3981. doi: 3910.1002/cncr.29596. Epub 22015 Aug 29519. [PubMed: 26287506] 
Kuntz G, Tozer JM, Snegosky J, Fox J, \& Neumann K (2014). Michigan Oncology Medical Home Demonstration Project: first-year results. Journal of Oncology Practice, 10(5), 294-297. doi: 210.1200/JOP.2013.001365. Epub 002014 Jul 001361. [PubMed: 24986111]

Liang H, Zhu J, Kong X, Beydoun MA, Wenzel JA, \& Shi L (2017). The Patient-Centered Care and Receipt of Preventive Services Among Older Adults With Chronic Diseases: A Nationwide Crosssectional Study. Inquiry, 54, 46958017724003. doi:10.1177/0046958017724003 [PubMed: 28814174]

McAneny BL (2013). The future of oncology? Come home, the oncology medical home. American Journal of Managed Care, 19(1 Spec No), cover, SP41-42.

McManus LS (2017). The Patient-Centered Medical Home and Diabetes Mellitus Outcomes: A Systematic Review. Retrieved from http://scholarworks.waldenu.edu/cgi/viewcontent.cgi? article $=4413 \&$ context $=$ dissertations.

Moher D, Liberati A, Tetzlaff J, Altman DG, \& Group P (2009). Preferred reporting items for systematic reviews and meta-analyses: the PRISMA statement. PLoS Medicine, 6(7), e1000097. doi:10.1371/journal.pmed.1000097 [PubMed: 19621072]

National Committee for Quality Assurance. (2011). NCQA Patient-Centered Medical Home 2011: Health Care That Revolves Around You. Retrieved from http://www.ncqa.org/Portals/0/Programs/ Recognition/2011PCMHbrochure_web.pdf

National Committee for Quality Assurance. (2013). Patient-Centered Specialty Practice Recognition. Retrieved from https://www.ncqa.org/Portals/0/Newsroom/2013/PCSP\%20Launch/PCSPR \%202013\%20White\%20Paper\%203.26.13\%20formatted.pdf.

National Cancer Institute. (2018). Cancer Statistics. Retrieved from https://www.cancer.gov/aboutcancer/understanding/statistics.

National Cancer Institute. (2016). Navigating Cancer Care. Retrieved from https://www.cancer.net/ navigating-cancer-care/older-adults/when-cancer-not-your-only-health-concern.

Patient-Centered Primary Care Collaborative (PCPCC). (2012). Pilots and Demonstrations, Joint Principles of the Patient-Centered Medical Home. Retrieved from http://www.pcpcc.net/content/ joint-principles-patient-centered-medical-home.

Reibling N (2016). The Patient-Centered Medical Home: How Is It Related to Quality and Equity Among the General Adult Population? Medical Care Research Review, 73(5), 606-623. doi:10.1177/1077558715622913 [PubMed: 26931123]

Rosenthal TC (2008). The Medical Home: Growing Evidence to Support a New Approach to Primary Care. Journal of American Board of Family Medicine, 21(5), 427-40.

Sprandio JD (2010). Oncology patient-centered medical home and accountable cancer care. Community Oncology, 7(12), 565-572.

StataCorp. (2011). Stata Statistical Software: Release 12. College Station, TX: StataCorp LP. van den Berk-Clark C, Doucette E, Rottnek F, Manard W, Prada MA, Hughes R, . . Schneider FD (2017). Do Patient-Centered Medical Homes Improve Health Behaviors, Outcomes, and Experiences of Low-Income Patients? A Systematic Review and Meta-Analysis. Health Services Research. doi:10.1111/1475-6773.12737

Sullivan DR, Ganzini L, Lapidus JA, Hansen L, Carney PA, Osborne ML, Fromme EK, Izumi S, \& Slatore CG (2018). Improvements in hospice utilization among patients with advanced-stage lung cancer in an integrated health care system. Cancer, 124(2), 426-433. [PubMed: 29023648]

Waters TM, Webster JA, Stevens LA, Li T, Kaplan CM, Graetz I, \& McAneny BL (2015). Community Oncology Medical Homes: Physician-Driven Change to Improve Patient Care and Reduce Costs. Journal of Oncology Practice., 11(6), 462-467. doi: 410.1200/JOP.2015.005256. Epub 002015 Jul 005228. [PubMed: 26220931]

Wender RC, Altshuler M (2009). Can the Medical Home Reduce Cancer Morbidity and Mortality. Primary Care, 36(4), 845-58. [PubMed: 19913189]

Wheeler SB, Kohler RE, Goyal RK, Lich KH, Lin CC, Moore A, . . Domino ME (2013). Is medical home enrollment associated with receipt of guideline-concordant follow-up care among lowincome breast cancer survivors? Annals of Oncology., 24(9), 2324-2329. doi: 2310.1093/annonc/ mdt2195. Epub 2013 May 2323. [PubMed: 23709173] 
Wilper AP, Woolhandler S, Lasser KE, McCormick D, Bor DH, \& Himmelstein DU (2008). A national study of chronic disease prevalence and access to care in uninsured U.S. adults. Annals of Internal Medicine, 149(3), 170-176. [PubMed: 18678844] 


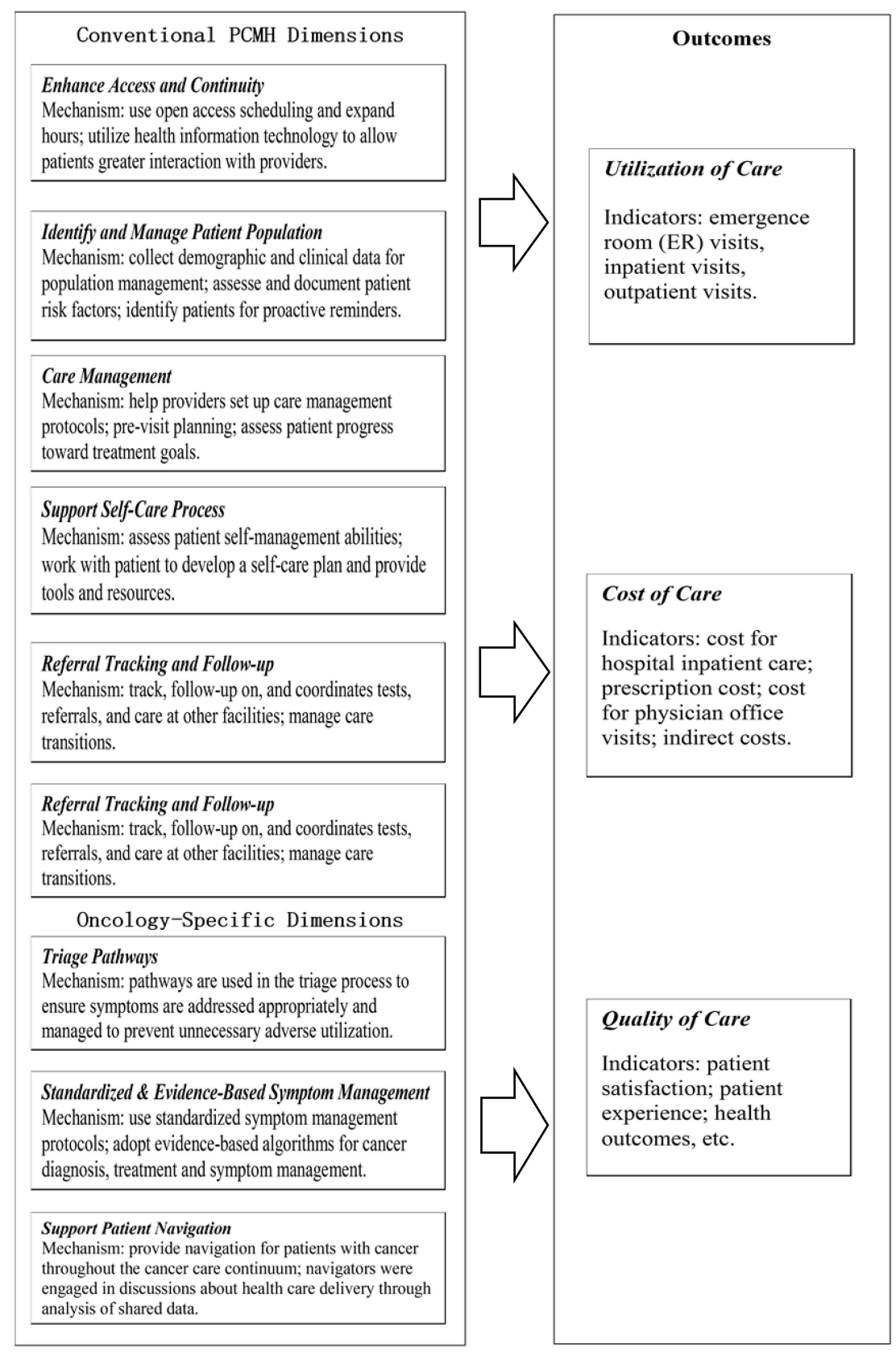

Note: the framework is adapted from the Joint Principles of the PCMH (PCPPC, 2012), and Wender and Altshuler (2009).

Figure 1.

Conceptual Framework for Patient-Centered Oncology Care 


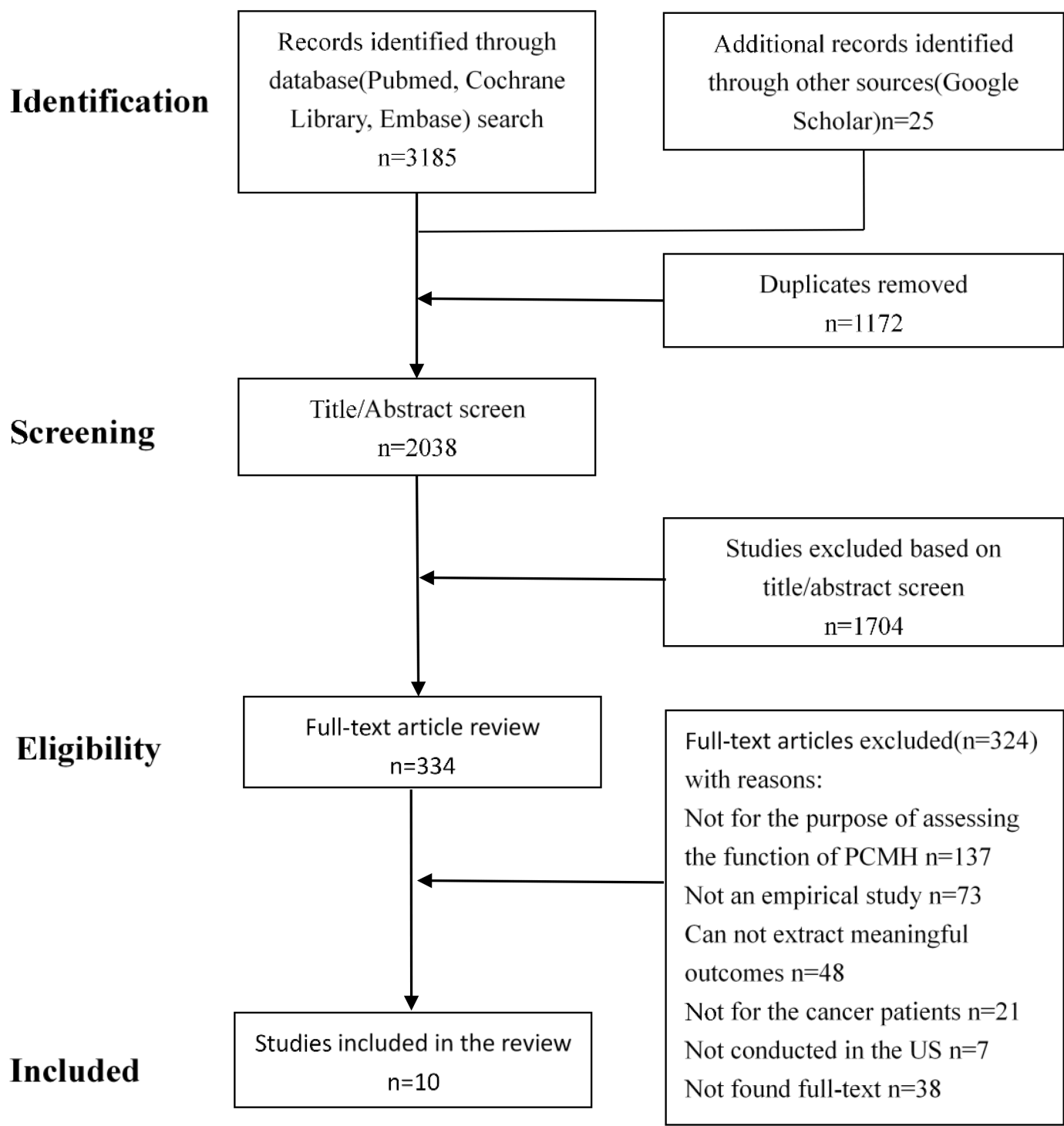

Figure 2.

Screening Process for the Eligible Studies 


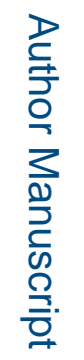

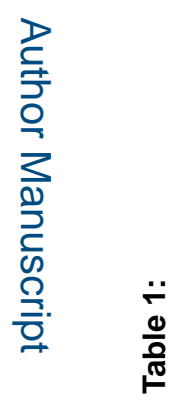

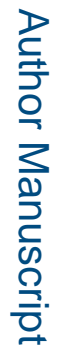

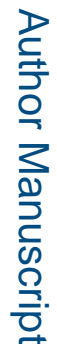

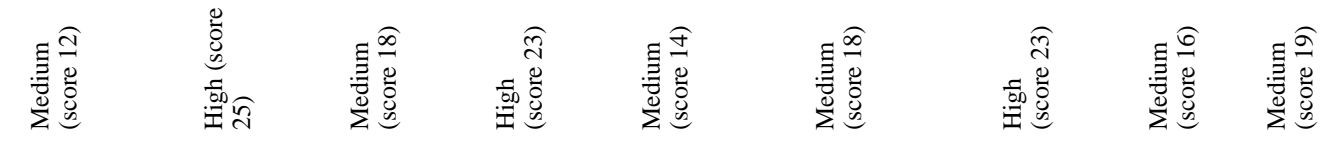

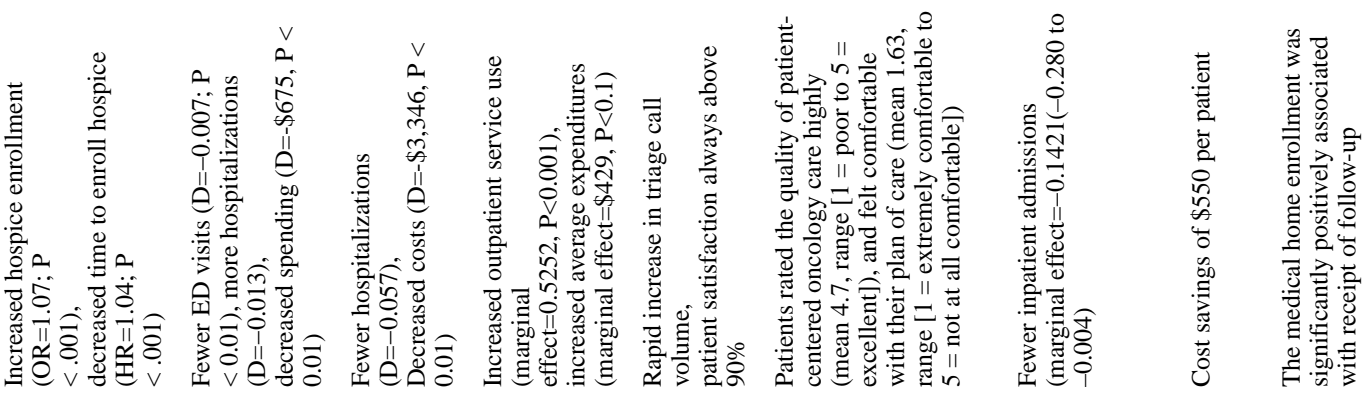

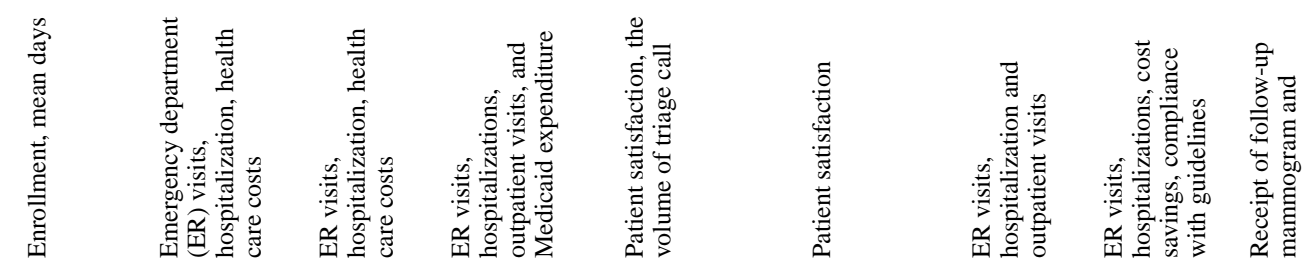

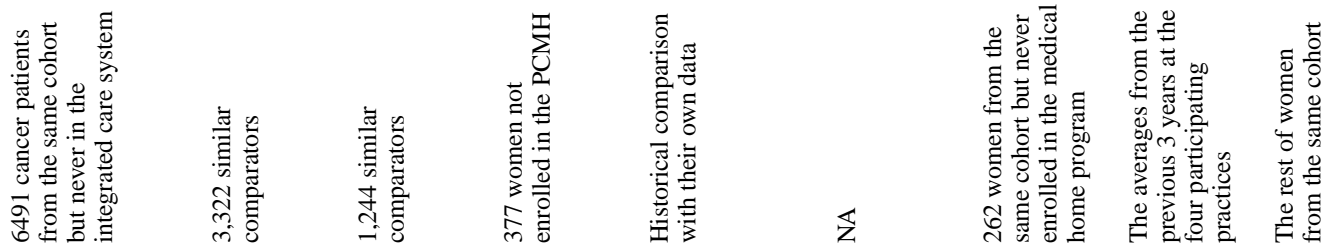

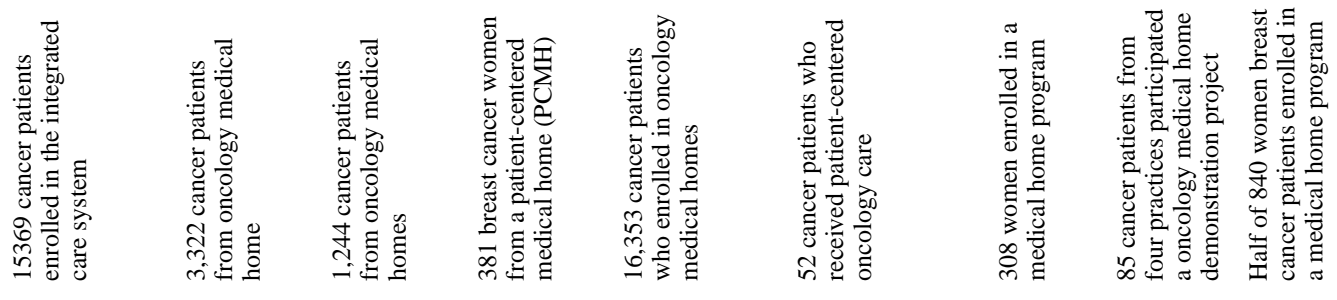

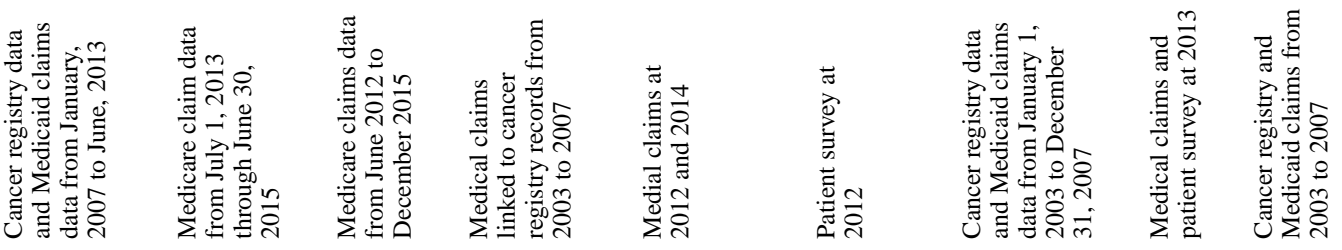

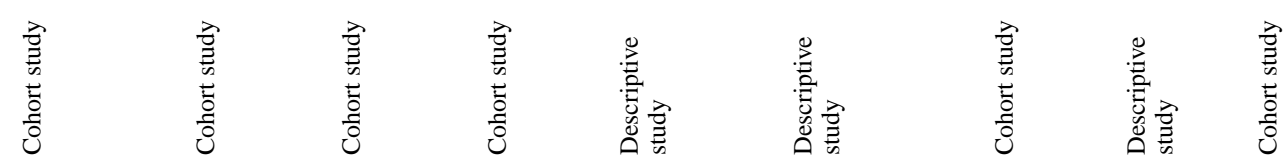

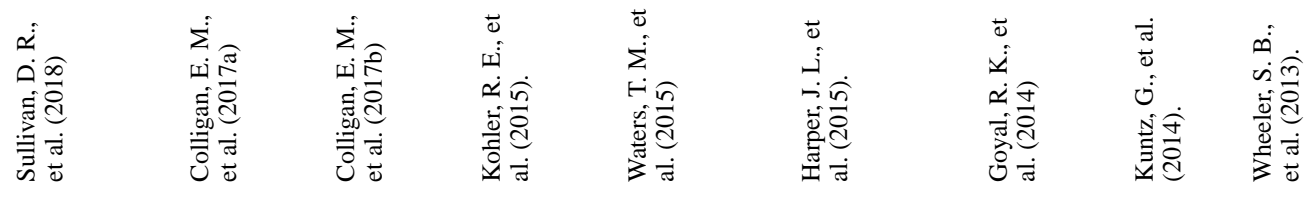
Health Care Manage Rev. Author manuscript; available in PMC 2021 October 01. 
Liang et al.

Page 19

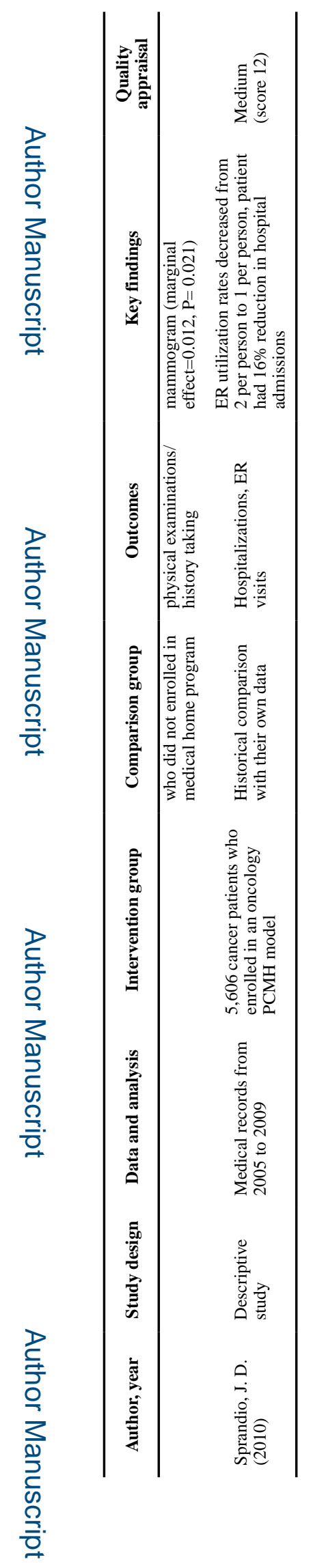

Health Care Manage Rev. Author manuscript; available in PMC 2021 October 01. 